\title{
Operaciones conjuntas: Operación Libertad para Iraky su comparación con la estrategia militar conjunta en Colombia*
}

\section{Joint Operations: Iraqi Freedom Operation and its Comparison with the Joint Military Strategy in Colombia}

\author{
Pedro Javier Rojas Guevara**
}

Recibido: 01/06/2015

Aprobado: 14/07/2015

Disponible en línea: 30/11/2015

\section{Resumen}

Ante el nuevo escenario de un postconflicto planteado en Colombia, las Fuerzas Militares adelantan un proceso de transformación y uno de los objetivos de este proceso es incrementar y perfeccionar las operaciones conjuntas, es decir, aquellas planeadas y ejecutadas por dos o más fuerzas. El primer paso se dio en 2003 con la creación de la Fuerza de Tarea Conjunta Omega, cuya base es la experiencia de las Fuerzas Armadas de Estados Unidos. Este artículo analiza la operación estadounidense Libertad para Irak -en especial el nivel estratégico y la unidad de mando- y la compara con los resultados alcanzados hasta ahora por Colombia en el campo de las operaciones conjuntas, donde la generación de sinergias y la flexibilidad operacional han aumentado la exactitud, la contundencia y la eficiencia de las Fuerzas Militares.

\section{Abstract}

In the newly proposed post-conflict scenario in Colombia, the Armed Forces are engaged in a process of transformation, and one of the objectives of this process is to increase and improve joint operations, that is, those planned and executed by two or more military forces. The first step towards this objective occurred in 2003 with the creation of the Omega Joint Task Force, based on the experience of the United States Armed Forces. This article analyzes the US Operation Iraqi Freedom -especially the strategic level and the unity of command- and compares it with the results achieved so far by Colombia in the field of joint operations, where the generation of synergies and operational flexibility have increased the accuracy, strength, and efficiency of the Military Forces.

doi:10.11144/Javeriana.papo20-2.ocol

\section{* Ensayo}

** Coronel del Ejército Nacional de Colombia. Magíster en Seguridad y Defensa Nacionales. Correo electrónico: pedrojrojasg@gmail.com 


\section{Palabras clave}

operaciones conjuntas; operación Libertad para Irak; doctrina; Ley de Seguridad Nacional (Colombia)

\section{Cómo citar este ensayo:}

Rojas-Guevara, P. J. (2015). Operaciones conjuntas: Operación Libertad para Irak y su comparación con la estrategia militar conjunta en Colombia. Papel Político, 2O(2), 613-623. http://dx.doi.org/10.11144/Javeriana.papo20-2.ocol

\section{Keywords}

joint operations; operation Iraqi Freedom; military doctrine; National Security Act (Colombia) 
El concepto de conjunción representa la verdad histórica de que ni el poder aéreo, ni el terrestre, ni el marítimo ganan guerras por sí solos.

Coronel ( $r$ ) Dennis M. Drew, Fuerza Aérea de los Estados Unidos

Adentrarnos en el análisis de la operación Libertad para Irak ejecutada por tropas pertenecientes a una coalición internacional liderada por el Ejército de Estados Unidos entre el 20 de marzo de 2003 y el 18 de diciembre de 2011, y su comparación con el concepto operacional conjunto en las Fuerzas Militares de Colombia que se originó en el 2003 con la activación de la Fuerza de tarea Conjunta Omega y marcó un hito en la forma de conducir militarmente las tropas, exige en primera instancia, definir de manera clara qué son las operaciones militares conjuntas.

Según el Diccionario de la guerra (Comunidad Astalaweb, s. f.) las operaciones conjuntas son "operaciones que pueden ser desarrolladas por una organización militar con componentes de dos o más fuerzas". Por su parte, el Curso de Orientación sobre Defensa Nacional (Codenal), desarrollado en la Escuela Superior de Guerra durante el segundo semestre de 2006, las define como:

[...] aquellas operaciones planificadas, sincronizadas y ejecutadas por organizaciones militares conjuntas, con componentes de dos o más Fuerzas, bajo la responsabilidad de un comandante, que se integran para cumplir una misión, con el fin de llevar a cabo un esfuerzo único y concentrado. (Flórez, 2012)

En efecto, las Fuerzas Armadas estadounidenses iniciaron la configuración de su doctrina conjunta a partir de la Segunda Guerra Mundial, mediante la alianza políticomilitar que la superpotencia materializó con la Unión Soviética y la Gran Bretaña. Precisamente, en 1947 se creó el Estado Mayor Conjunto (The Joint Chiefs of Staff) a través de la Ley de Seguridad Nacional, documento estratégico avalado por el presidente Harry S. Truman, cuyo propósito principal fue reformar la organización de las Fuerzas Militares y la comunidad de inteligencia al finalizar la gran confrontación bélica. Dicha transformación castrense, que posteriormente tuvo algunos ajustes, es lo que hoy conocemos en gran medida como el Departamento de Defensa de Estados Unidos.

Igualmente, la Ley Nacional de Seguridad estableció el Consejo de Seguridad Nacional, que se erigió como una gran central de coordinación para los temas relacionados con las políticas de seguridad y defensa nacionales. Así mismo, se creó la Agencia Central de Inteligencia (CIA), primera central de espionaje instituida en tiempos de paz. 
En suma, Estados Unidos impulsó significativamente su gran capacidad y poderío militar, lo cual ha posicionado a esta nación como líder en operaciones conjuntas, entre las que se destaca especialmente la operación Overlord, más conocida como el Desembarco de Normandía, desarrollada el 6 de junio de 1944 en el ocaso de la Segunda Guerra Mundial, dirigida por el General Dwigth Eisenhower, quien tuvo bajo su mando fuerzas navales, fuerzas de tierra durante la fase de asalto, fuerzas de bombarderos estratégicos del Reino Unido y dos fuerzas aéreas tácticas, la novena Fuerza Aérea de los Estados Unidos y la segunda Fuerza Aérea Británica, Royal Air Force (RAF). El Día D, como se conoce también al Desembarco de Normandía, pasó a la historia como una de las mayores demostraciones de despliegue militar conjunto con un resultado políticoestratégico contundente que marcó el comienzo del fin para Hitler y su Tercer Reich.

Sir Winston Churchill, refiriéndose a la compleja tarea de que las tres fuerzas -Ejército, Armada y Aviación- que interactuaron durante el entrenamiento, con miras a la ejecución de la operación Overlord, afirmó:

Hacía tiempo que el Estado Mayor de Operaciones Conjuntas dirigido por el almirante Mountbatten, cuyo sucesor fue el general Laycock, había fijado la teoría y la práctica de las operaciones anfibias. Ahora había que enseñárselas a todos los participantes, además del intenso entrenamiento general necesario para la guerra moderna que, evidentemente, se estaba llevando a cabo hacía tiempo en Gran Bretaña y en Estados Unidos mediante grandes y pequeñas maniobras con fuego real. Muchos oficiales y soldados entraron en combate por primera vez, pero todos se comportaron como militares expertos. (2012, p. 859)

\section{Operación Libertad para Irak}

En el análisis de esta operación es importante destacar el principio de guerra llamado Unidad de Mando ${ }^{1}$ como factor determinante en la conducción del concepto operacional conjunto. Por ello, en lo atinente a su estructura de mando, la operación Libertad para Irak contó con un esfuerzo principal por parte de las Fuerzas Armadas estadounidenses, en el cual se integraron las fuerzas británicas, sin embargo, en los niveles de mando, los generales estadounidenses estuvieron en el primer orden. Por tanto, quien fungió como presidente de la Junta de Jefes de Estado Mayor fue el General Myers, que también se desempeñó como principal asesor militar directo del presidente de Estados Unidos a través del Consejo de Seguridad Nacional, no obstante, este oficial no estaba dentro de la línea de mando operacional.

\footnotetext{
1 "La decisiva aplicación del poderío total solo se obtiene con la unidad de mando" (Fuerzas Militares de Colombia, 1997, p. 18). La unidad de mando debe reflejarse en el esfuerzo conjunto, y este requiere la indicación de un solo propósito, la cooperación y la coordinación de todos los elementos y acciones de las fuerzas constituidas. En otras palabras, se requiere trabajo en equipo.
} 
A nivel estratégico, a cargo del Pentágono para la zona de Oriente Próximo, el Mando Estratégico Central (Centcom) estuvo bajo la tutoría del General del Ejército Tommy Franks, también comandante de las tropas de la coalición y que tenía a su disposición cuatro componentes (terrestre, marítimo, aéreo y de operaciones especiales). Este audaz militar ejerció el mando de manera sencilla e inteligente y una de las claves de su éxito fue la excelente dupla que hizo con el Secretario de Defensa de los Estados Unidos, Donald Rumsfeld, quien lo respaldó y empoderó para desarrollar una campaña militar con total autonomía y poder de decisión, que a la postre terminó con el cumplimiento perfecto de la misión, es decir, la derrota de las fuerzas iraquíes y el consecuente derrocamiento de Saddam Hussein.

El buen general Franks, en su obra American Soldiers, narra sobre la operación Libertad para Irak y su carácter conjunto lo siguiente:

En la provincia de Patkia el otro día, soldados, Navy Seals, tripulaciones de la Fuerza Aérea, marines pilotos de los cobra y Bert Calland y Dell Dailey's de las Fuerzas Especiales, combatían, morían y daban bajas a Al Qaeda. No había campañas terrestres, campañas aéreas o campañas de fuerzas especiales. Había un solo plan integrado, y nuestra gente combatía como una fuerza conjunta. (2005, p. 383)

Sin lugar a dudas, el factor determinante para la victoria en esta operación fue la unidad de mando y un plan militar muy bien diseñado, con unidades altamente preparadas y entrenadas, con equipos y armas de última generación, con fuerzas especiales debidamente equipadas y con una elevada moral. Además, las tropas de la coalición se distinguían por su excepcional carácter disuasivo y polivalente, lo que garantizó el desarrollo de operaciones terrestres (ofensivas, defensivas, de estabilidad y de apoyo) mediante maniobras de armas combinadas, fuerzas especiales y seguridad en área extensa, con una armónica integración de los medios informáticos y de comunicaciones con todos sus servicios, que se conjugaron hábilmente por medio de la interoperabilidad.

Efectivamente, el mando militar estadounidense basó los lineamientos de la operación en las lecciones aprendidas del pasado, fundamentadas en serios análisis realizados por oficiales idóneos y con gran experiencia en las guerras de la década de los noventa. En palabras del General Myers, presidente de la Junta de Jefes de Estado Mayor estadounidense (JEMAD) durante la operación Libertad para Irak: "El combate conjunto es la clave para lograr grandes éxitos en el campo de batalla” (Ceseden, 2003, p. 59).

Es así como el Ejército de los Estados Unidos ha tenido en los últimos 25 años importantes cambios en su doctrina y organización conjunta. El último de ellos comenzó con una conferencia realizada el 11 de octubre de 2011 y se espera que culmine a finales de 2015. Pero sin duda, el proceso más recordado fue el de reconstrucción del 
Ejército que surgió una vez culminada la Guerra Fría. Este salto generó una soberbia estructura militar profesional que triunfó de manera decisiva en la Guerra del Golfo (1991), que antecedió a la operación objeto de este estudio y que en la actualidad es modelo y referente en todo el mundo.

$\mathrm{Al}$ respecto, el General Sullivan, de Estados Unidos, aseveró

Hoy, el Ejército necesita ser lo bastante flexible para tener éxito, no solo en la guerra tradicional, sino en nuevas misiones como mantener la paz, suministrar ayuda humanitaria y alivio en los desastres, y construir la democracia, tanto en casa como en el extranjero" (Sullivan \& Harper, 1998, p. 17)

Estas palabras coinciden hoy por hoy con la expectativa del ejército del futuro en Colombia. Parafraseando a Sullivan, los colombianos son nuestros clientes y aunque no estamos en peligro de perderlos, el peligro está en fallarles en el campo de combate, en la guerra contra las organizaciones terroristas y criminales (Sullivan \& Harper, 1998, p. 20).

El Centro Superior de Estudios de la Defensa Nacional del Reino de España (Ceseden), en una de sus monografías, hizo un minucioso y acertado análisis de los aspectos diferenciales de la campaña militar del 2003 sobre Irak, entre los cuales destaca:

- Suplir la menor cantidad de fuerzas disponibles en el teatro por una mayor letalidad y efectividad, logradas a través de innovaciones tecnológicas y doctrinales.

- Intensificar en el tiempo y en la amplitud de sus objetivos los bombardeos aéreos estratégicos y en apoyo a las operaciones terrestres, a través de un empleo masivo de los misiles de crucero y de las municiones inteligentes.

- Mantener la firme voluntad de empleo de fuerzas terrestres, que invadirían el país llegado el caso.

- Buscar en todo momento la colaboración de las Fuerzas Armadas y población civil opuesta a Sadam Husein, de forma que contribuya a la desestabilización interna del régimen.

- Dirigirse directa y rápidamente a los objetivos finales, mediante un avance continuado de las fuerzas terrestres, evitando enfrentamientos directos con unidades enemigas en la medida de lo posible y sobre todo, al interior de las ciudades.

- Permitir que las fuerzas convencionales y especiales, las de operaciones aéreas y terrestres, las de agentes y medios de inteligencia, etc., ejerzan su acción de manera simultánea. Todo ello integrado mediante un sistema de mando y control fiable y seguro, que aporte una elevada capacidad de combate de forma permanente. En definitiva, se puede decir que nos encontramos ante una forma de hacer la guerra que conjuga elementos audaces y novedosos con otros más tradicionales y seguros. (2003, p. 59) 
En conclusión, las Fuerzas Armadas de los Estados Unidos siguen a la vanguardia de los ejércitos del mundo y por lo tanto, se constituyen en referente doctrinal y de organización conjunta para Colombia. Por cierto, el documento intitulado: "Visión Conjunta 2020: Las Fuerzas Armadas de los Estados Unidos preparándose para el futuro" escrito por el Jefe de Estado Mayor Conjunto en el año 2000 y reproducido un año más tarde por la revista Military Review, sentencia de manera clara lo siguiente acerca de la importancia del Comando y Control como eje de las operaciones conjuntas:

El comando y control es el ejercicio de la autoridad y dirección de las actividades de la fuerza conjunta. Es necesario para la integración de las competencias centrales de las instituciones individuales en las operaciones conjuntas eficaces. La creciente importancia de los aspectos de las operaciones multinacionales e interagenciales complican e intensifican el desafío de realizar la integración. El comando y control incluye el planeamiento, dirección, coordinación y control de las fuerzas y operaciones con enfoque en la ejecución eficaz del plan operativo; pero su función central es la toma de decisiones. (Escuela de Comando y Estado Mayor, 2001, p. 18)

\section{El concepto operacional conjunto en Colombia}

En 2002 el Gobierno colombiano encabezado por el Presidente Álvaro Uribe Vélez, emitió sus directrices gubernamentales en materia de seguridad y defensa mediante la "Política de Defensa y Seguridad Democrática”. Basándose en dicha política, las Fuerzas Militares colombianas, asesoradas por sus homólogas estadounidenses y encabezadas por el Ejército en coordinación con la Policía Nacional, diseñaron sus planes respectivos. Así nació en 2003 la primera Fuerza de Tarea Conjunta, llamada Omega, con una misión puntual: neutralizar la evolución del plan estratégico de las FARC en los departamentos del Meta, Guaviare y Caquetá, región considerada como la retaguardia estratégica del grupo criminal. El experto en Estudios Políticos y Relaciones Internacionales Javier Flórez Henao afirma en uno de sus escritos sobre el reciente enfoque doctrinal:

El surgimiento de las unidades conjuntas no es solo el producto de la experiencia e influencia norteamericana, es el resultado también de experiencias propias en combates caracterizados por la variada geografía colombiana que permitieron tomar a esta forma de organización militar como una posible solución para hacer más efectivo el despliegue de las tropas; y así mismo, generar una gran concentración del poder militar, unidad de esfuerzo, sincronización, sinergia y flexibilidad en las Fuerzas Militares. (2012, p. 68)

En cuanto a la organización de carácter conjunto, las Fuerzas Militares de Colombia, desde la década anterior, cuentan con comandos conjuntos establecidos de la mano del Ejército Sur de los Estados Unidos y en los últimos años se ha dado paso a organizaciones 
provisionales, denominadas Fuerzas de Tarea Conjunta, como parte de la nueva estrategia de lucha contra el terrorismo, el narcotráfico y los grupos armados ilegales.

Esta propuesta de organización conjunta fue desarrollada por los Comités de Revisión Estratégica e Innovación (CREI) desde el año 2011 y tiene como base la experiencia de las Fuerzas Armadas estadounidenses, puntualmente la obtenida en la operación Libertad para Irak, y los buenos resultados alcanzados por la Fuerza de Tarea Conjunta Omega, pionera en esta forma de operar entre fuerzas. Dichos resultados obedecen entre otras razones, a la generación de sinergias que incrementan la exactitud, la contundencia y la eficiencia operacional.

De igual forma, el Comité de Diseño del Ejército del Futuro (CEDEF) contó con la asesoría de reconocidos oficiales del Ejército de los Estados Unidos, algunos de los cuales participaron en la guerra de Irak, y civiles expertos en trasformación como Paul Davis ${ }^{2}$, traídos a Colombia para coadyuvar en el objetivo de garantizar las capacidades estratégicas para la Defensa Nacional. Dichos académicos y militares se basaron en las experiencias y ejecutorias de brillantes generales como Norman Schwarzkopf (1934-2012), Comandante en Jefe del Mando Central de los Estados Unidos y Comandante de las Fuerzas de la Coalición en la Guerra del Golfo de 1991; y como John Philip Abizaid (1951, de origen árabe libanés cristiano), Comandante del Comando Central de los Estados Unidos entre 2003 y 2007, quien tomó el liderazgo de las fuerzas de ocupación en Irak y Afganistán al retiro del General Franks. Basados en las teorías y análisis de los autodenominados NeoLidell Hart ${ }^{3}$, ellos aplicaron el modelo alemán (maniobra de armas combinadas o doctrina acorazada $^{4}$ ) en las guerras de Irak de 1991 y 2003, respectivamente.

En el caso colombiano, este modelo de guerra conjunto es adaptable y aplicable en los escenarios donde se presente una amenaza externa que cuente con material bélico de última generación. Sin embargo, como toda política de cambio en la organización, su éxito es contingente, en la medida en que responde a exigencias del ambiente operacional en un lapso determinado. Basándose en el concepto de organización modular, las Fuerzas de Tarea Conjunta precisan de flexibilidad para ser eficaces, viables y adecuadas, frente a cualquier tipo de cambio o reto.

\footnotetext{
${ }^{2}$ Experto en transformación de la corporación Research and Development (RAND), asesoró al Ejército de los Estados Unidos.

${ }^{3}$ Grupo de ofíciales estadounidenses que adaptaron las enseñanzas de Lidell Hart y posteriormente las aplicaron a las guerras modernas. Entre ellos se destacó Omar Bradley, último general de cinco estrellas de Estados Unidos, quien participó en la Segunda Guerra Mundial. Fue uno de los más destacados comandantes aliados y dirigió con éxito numerosas operaciones conjuntas.

${ }^{4}$ Se entiende como "la habilidad, idoneidad y pericia que permite el uso de los elementos de potencia de combate en una acción sincronizada, plenamente integrada, con el propósito de obtener y explotar la iniciativa, para derrotar fuerzas terrestres, obtener, ocupar y/o recuperar terreno y lograr ventajas físicas, temporales y psicológicas sobre el enemigo" (Ejército Nacional \& Ejército de Chile, 2014, p. 5).
} 
Ante el nuevo escenario planteado en Colombia, es decir, un postconflicto, es un error continuar con la idea de organizar fuerzas de tarea de armas combinadas, toda vez que estas unidades son eminentemente transitorias y creadas para un fin y una misión específicos. Culturalmente el Ejército colombiano ha afrontado las contingencias operacionales dentro del conflicto armado interno haciendo uso de ellas; en efecto, la columna vertebral del Plan Espada de Honor (estrategia militar que adelantan las Fuerzas Militares de Colombia desde el año 2011) son las Fuerzas de Tarea.

No obstante, en el postconflicto y ante un potencial - pero poco probable- escenario de guerra regular planteado, se deben organizar e implementar las Brigadas de Armas Combinadas en el Teatro Norte y de acuerdo con la situación, actuar con fuerzas de tarea o equipos de combate (este tipo de unidades se caracteriza por su poder de combate).

Es menester, para efectos de organización de las unidades, remitirnos a la sociología militar y también a la polemología, para comprender qué es la organización operativa, entendida como la estructura funcional que deben adoptar las unidades de un ejército y las instrucciones (tácticas y estrategias) para cumplir la misión. Precisamente, el profesor chileno Omar Gutiérrez Baldebenito, en su obra Sociología militar. La profesión militar en la sociedad democrática (2002), afirma: "La eficacia de las organizaciones operativas radica en la existencia de una doctrina y en la cohesión de la fuerza. El éxito de las organizaciones operativas descansa en los valores morales de los hombres que integran la fuerza” (p. 189). He aquí los preceptos fundamentales de cualquier ejército victorioso.

Para terminar, es necesario señalar que las Fuerzas Militares de Colombia adelantan un proceso de transformación en cuanto a su organización y doctrina, como se indicó en párrafos anteriores, paralelo a unas conversaciones de paz que lleva a cabo el Gobierno con el grupo terrorista de las FARC. En tal sentido, le corresponde al Alto Mando Militar tomar decisiones apropiadas y convenientes en lo referente a la adquisición de armas y equipos y a la organización de unidades preparadas para la ejecución de operaciones conjuntas efectivas responsables y que le apunten a la seguridad nacional, definida en el Manual de defensa y seguridad nacional FF. MM. 3-43 como "la situación en la cual los intereses de la Nación se hallan a cubierto de interferencias y perturbaciones substanciales” (Fuerzas Militares de Colombia, 1996, p. 21).

En consecuencia, las operaciones conjuntas -en plena progresión en Colombiasiempre deberán estar alineadas con la doctrina conjunta aplicable y vigente para la operatividad de las Fuerzas Militares:

La defensa nacional se presenta dentro de un concepto dinámico, porque implica una constante actividad de previsión y resistencia frente a los ataques y amenazas de que pueda ser objeto la seguridad del país. Concebir la Defensa Nacional como la adopción de 
una actitud pasiva o de simple expectativa ante hechos o situaciones que atentan contra esa seguridad equivale a la aceptación anticipada de la derrota. (Fuerzas Militares de Colombia,1996, p. 25)

\section{Conclusiones}

- El concepto operacional conjunto es antiguo en el mundo, pero novedoso para nuestro país y sin duda exige una coherencia política y por ende, militar, en el sentido de determinar cuál es la prioridad en términos de seguridad y defensa para Colombia en esta coyuntura política.

- Esta construcción doctrinaria y de organización conjunta deberá reducir la incertidumbre, es decir, debe ser prospectiva y enfocarse en los escenarios futuros. De igual forma, debe estar alineada con la dinámica del contexto global y ser moderna, vigente y fácilmente adaptable a las necesidades de la fuerza para combatir las nuevas amenazas. En una frase del Mayor General Alberto Mejía Ferrero, la doctrina y la organización conjunta deben "pintar el Ejército y las demás fuerzas de un solo color" (2012).

- La defensa de la soberanía requiere un concepto operacional conjunto, mediante métodos disuasivos para enfrentar una amenaza externa que pueda buscar la secesión de un terreno de nuestro país; por esta razón, se deben tener sistemas de armas que garanticen la defensa, puedan neutralizar fuerzas invasoras y estén en capacidad de pasar de la defensiva a la ofensiva para poder ganar la ventaja militar (sistema de armas antitanques y de ingenieros).

- La organización militar conjunta requiere, para la adquisición de sistemas de defensa, conocer la amenaza -sus capacidades de maniobra, así como su apoyo al combate y su apoyo de servicios para el combate-, para determinar el tipo de arma que se requiere; por ejemplo un T-9o se puede destruir con otro tanque o con un antitanque de alcance superior a $4 \mathrm{~km}$. Los trenes logísticos del enemigo pueden ser penetrados en profundidad para destruirlos y dejar totalmente aisladas a las unidades que apoyan.

- Las operaciones conjuntas en Colombia deben estar enmarcadas en lo preceptuado en la doctrina conjunta contenida en el Manual de seguridad y defensa nacional: "La Defensa Nacional no es otra cosa que el medio de que se vale el Estado para lograr uno de sus más importantes fines: la seguridad" (Fuerzas Militares de Colombia, 1996, p. 25).

- Durante los últimos 50 años, las Fuerzas Militares de Colombia han desarrollado la mayor parte de su doctrina y organización en función de las demandas de un conflicto interno con matices de guerra contrainsurgente (amenaza interna) y tomando como principal referente en guerra de guerrillas a las Fuerzas Armadas de los Estados Unidos. Debido a ello, se relegó a un segundo plano el desarrollo doctrinal y organizacional conjunto característico de la mayoría de los ejércitos, basado en la defensa nacional frente a amenazas externas; esto ha llevado a que la institución se 
aleje de manera sustancial de su función más importante: la defensa nacional en el contexto de la doctrina regular.

\section{Referencias}

Centro Superior de Estudios de la Defensa Nacional (Ceseden) (2003). Estudio preliminar de la operación Libertad para Irak. Madrid: Ministerio de Defensa, Secretaría General Técnica.

Churchill, W. (2012). La segunda guerra mundial. Buenos Aires: Editorial El Ateneo. Comunidad Astalaweb. (s.f.). Diccionario de la guerra. Diccionarios en la red. Recuperado de http://diccionarios.astalaweb.com/Local/Diccionario\%2ode\%2oguerra.asp

Drew, D. M. (o9 de agosto de 1990). El mundo se ve distinto a 10.000 pies de altura. Air \& Space Power Journal. Recuperado de http://www.airpower.maxwell.af.mil/ apjinternational/apj-s/1990/1trimes9o/drew.html

Ejército Nacional de Colombia y Ejército de Chile (2014). Informe de Adecuación de la Doctrina Acorazada del Ejército de Chile al Comandante del Ejército de Colombia. Bogotá: Ejército Nacional.

Escuela de Comando y Estado Mayor (2001). Visión Conjunta 2020: Las Fuerzas Militares de los EE. UU. preparándose para el futuro. Military Review, (12), 3-21.

Flórez, J. A. (2012). La doctrina conjunta en Colombia: análisis de la Fuerza de Tarea Conjunta Omega. Tesis de Maestría. Bogotá, Colombia: Universidad Nacional. Recuperado de http://www.bdigital.unal.edu.co/7724/1/699243.2012.pdf

Franks, T. (2005). American soldier. New York: Harper Collins Publishers.

Fuerzas Militares de Colombia (1996). Manual de seguridad y defensa nacional. FF. MM. 3-43. Reservado. Bogotá: FF. MM.

Fuerzas Militares de Colombia (1997). Manual de estrategia militar general. FF. MM. 3-4. Reservado. Bogotá: FF. MM.

Gutiérrez, Ó. (2002). Sociología militar. La profesión militar en la sociedad democrática. Santiago de Chile: Editorial Universitaria.

Mejía, A. (2012). Conferencia ante oficiales y suboficiales de la Jefatura de Doctrina sobre transformación del Ejército. Bogotá: Teatro Patria.

Sullivan, G., \& Harper, M. (1998). La esperanza no es un método. Bogotá: Norma. 